\title{
Control Unit for Electromagnet Current
}

\author{
G. A. Belov ${ }^{1}$, S. V. Abramov ${ }^{1}$, G. V. Malinin, ${ }^{1, *}$, and A. V. Salov ${ }^{2}$ \\ ${ }^{1}$ I. N. Ulyanov Chuvash State University, Cheboksary, Russia \\ ${ }^{2}$ Relematika Ltd, Cheboksary, Russia
}

\begin{abstract}
The paper deals with the results of research and development of the electromagnet current control unit for the case when only two outputs without specifying the polarity of the source connection may be available from a circuit with a series of DC voltage sources and electromagnet winding. Theoretical results have been verified by modelling in Matlab-Simulink environment as well as by testing the starter of the contactor air switch.
\end{abstract}

\section{Introduction}

Today, electronic blocks for optimal control of their responding, holding and switching-out processes are widely used by the world's leading manufacturers of electromagnetic devices (relays, contactors, air- and oilcircuit breakers) [1]. However, they haven't been applied extensively in Russian-manufactured devices. The winding of the electromagnet that drives a moving element of the device is the load of such electronic blocks. Western manufacturers develop a wide range of monolithic integrated circuits (IC) for microprocessor control of the coil current, ranging from control windings for hammers of matrix printers [2] to electromagnet windings for high-power electromagnetic devices [3-8]. There is an extensive literature on the development of the electromagnet current control units [9-15]. However, it is assumed in all these papers that there may be outputs from the winding constant voltage source as well as from the winding itself.

The paper presents the results of the development of the electromagnet current control unit (ECCU) for the case when only two outputs, i.e. terminal outputs of the series circuit, from the open circuit with DC power supply connected in series are available. Moreover, voltage polarity at terminals of the circuit has not been present.

\section{Functional scheme of a switching unit}

Figure 1 shows ECCU main circuit consisting of three power semiconductor keys with drivers Dr and current sensor (CS). A diode bridge may be switched on at ECCU input to avoid incorrect connection of ECCU to the electromagnet coil. Transistor VT1 is designed to connect the electromagnet coil $L$ to the DC voltage source $E$ when the electromagnetic device is switched on. To quickly reduce current $i_{L}$ in the winding when the electromagnetic device is switched on, transistor VT1 is locked, but transistor VT2 allowing transferring the energy accumulated in the coil to capacitor $C$ at capacitor charging intervals $t_{\mathrm{ch}}$ is unlocked. To provide discharge of the energy accumulated in the capacitor to resistor $\mathrm{R}$ when it is charged up to the threshold voltage $U_{\text {th2 }}>\mathrm{E}$, the capacitor is periodically discharged by repetitive unlocking transistor VT3 for discharging time $t_{\mathrm{dis}}$ and locking it for time $t_{\mathrm{ch}}$. To reduce current $i_{L}$ rather quickly, the average voltage at capacitor $U_{\text {Cav }}$ should be higher than supply voltage $E$ by some value.

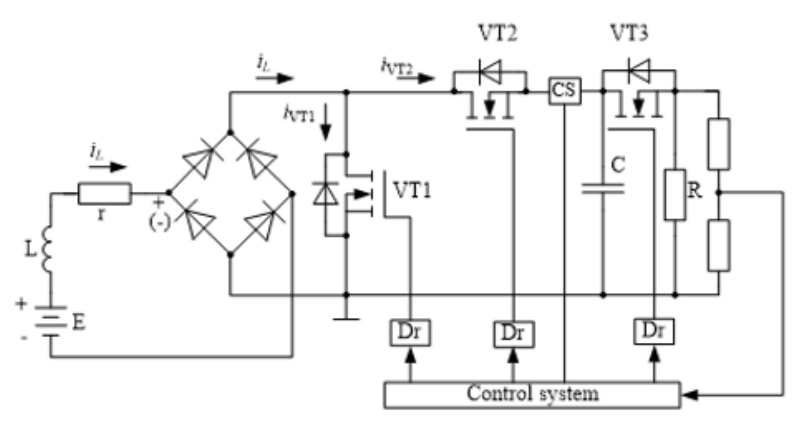

Fig. 1. A scheme for the high power part of the electromagnet control unit.

\section{Calculated ratios}

Calculated ratios for the capacitor charging-discharging periodic process could be obtained by the averaging method. True equation written as.

$$
C \frac{d u_{C}}{d t}=\left\{\begin{array}{l}
i_{L}, 0 \leq t \leq t_{\mathrm{ch}}, \\
i_{L}-\frac{u_{C}}{R} \quad 0 \leq t \leq t_{\mathrm{dis}},
\end{array}\right.
$$

is integrated for the time $t_{\mathrm{ch}}+t_{\mathrm{dis}}$, and the following equation could be obtained

$$
C \Delta u_{C}\left(t_{\mathrm{ch}}+t_{\mathrm{dis}}\right)=\int_{0}^{t_{\mathrm{ch}}+t_{\mathrm{dis}}} i_{L} d t-\frac{1}{R} \int_{t_{\mathrm{ch}}}^{t_{\mathrm{ch}}+t_{\mathrm{dis}}} u_{C} d t,
$$

\footnotetext{
* Corresponding author: malgrig6@mail.ru
} 
where $\Delta u_{C}\left(t_{\mathrm{ch}}+t_{\mathrm{dis}}\right)$ is voltage increment $u_{C}$ for the time $t_{\mathrm{ch}}+t_{\text {dis. }}$.

In our case, $\Delta u_{C}\left(t_{\mathrm{ch}}+t_{\mathrm{dis}}\right)=0$, so equation (1) could be written as follows:

$$
i_{\text {Lav }}-\frac{1}{R} \frac{t_{\mathrm{dis}}}{t_{\mathrm{ch}}+t_{\mathrm{dis}}} u_{\mathrm{Cav}}=0
$$

where average current $i_{L}$ and average voltage $u_{C}$ are

$$
i_{\text {Lav }}=\frac{1}{t_{\mathrm{ch}}+t_{\mathrm{dis}}} \int_{0}^{t_{\mathrm{ch}}+t_{\mathrm{dis}}} i_{L} d t, u_{\mathrm{Cav}}=\frac{1}{t_{\mathrm{ch}}+t_{\mathrm{dis}}} \int_{0}^{t_{\mathrm{ch}}+t_{\mathrm{dis}}} u_{C} d t
$$

Besides, it is accepted that

$$
\int_{t_{\mathrm{ch}}}^{t_{\mathrm{ch}}+t_{\mathrm{dis}}} u_{C} d t=t_{\mathrm{dis}} u_{C \mathrm{av}}
$$

Charging time $t_{\mathrm{ch}}$ could be determined by the following formula

$$
t_{\mathrm{ch}}=\frac{C \Delta u_{C}}{i_{L \mathrm{av}}}
$$

where the value $\Delta u_{C}=U_{\text {th2 }}-U_{\text {th1 } 1}$ may be prescribed, assuming that current change $i_{L}$ for the time $t_{\mathrm{ch}}$ is small.

The value $t_{\text {dis }}$ could be expressed through $t_{\mathrm{ch}}$ from equation (2) as follows:

$$
t_{\mathrm{ch}}=\frac{C \Delta u_{C}}{u_{C \mathrm{av}} / R-i_{L \mathrm{av}}}
$$

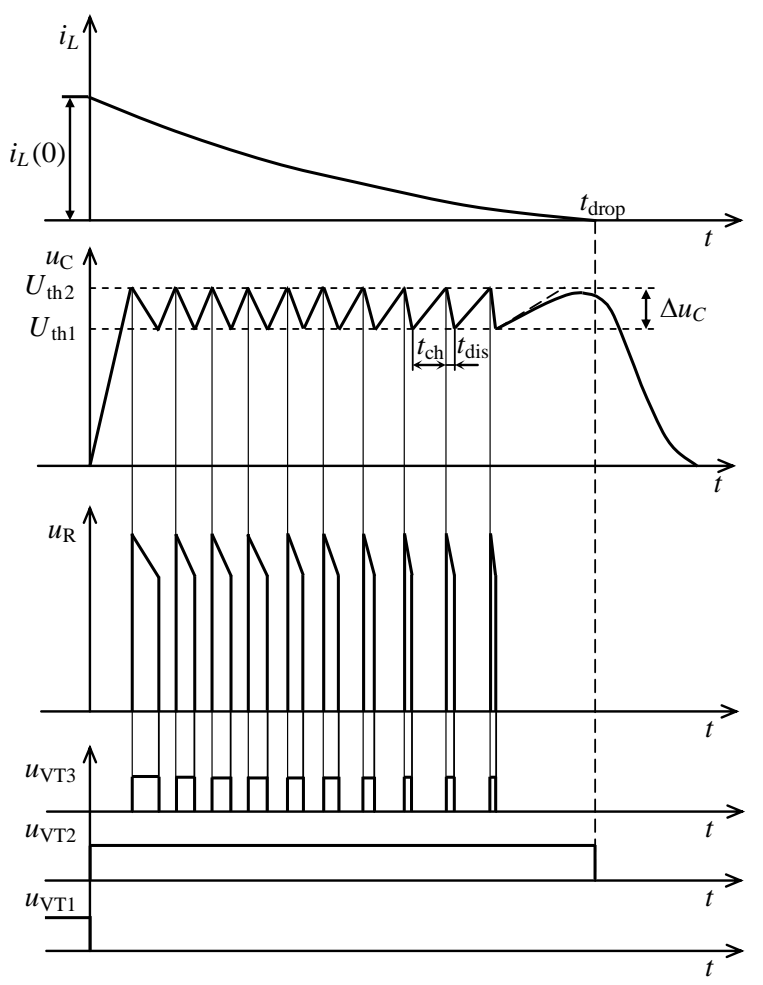

Fig. 2. Time charts for the process of discharging the energy accumulated in the coil.
Then, the duration of one capacitor chargingdischarging cycle could be determined by the following expression:

$$
t_{\mathrm{ch}}+t_{\mathrm{ch}}=\frac{C \Delta u_{C} u_{C \mathrm{av}}}{R i_{\text {Lav }}\left(u_{C \mathrm{av}} / R-i_{\text {Lav }}\right)} .
$$

The formula (5) error may increase with reduction of current $i_{L}$, since the charging time increases excessively. Figure 3 shows the theoretical calculated dependencies of the capacitor charging and discharging intervals.

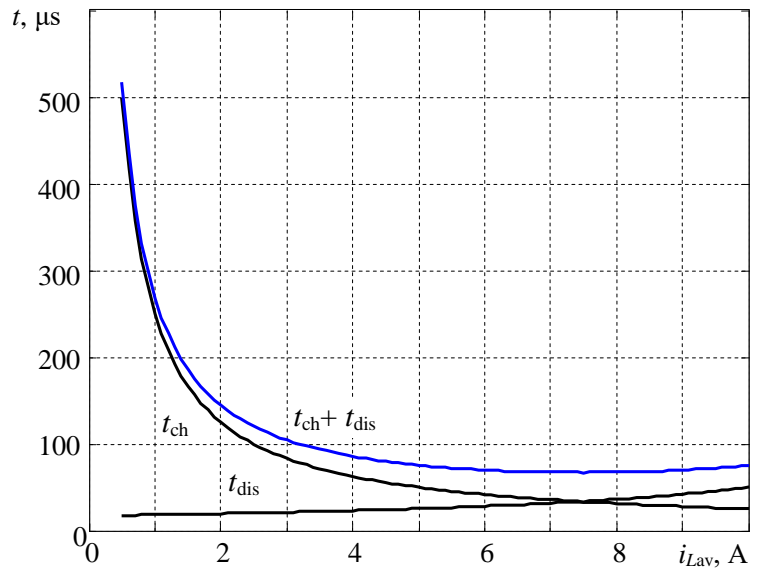

Fig. 3. Calculated curve for dependencies of the capacitor charging $t_{c h}$ and discharging $t_{d i s}$ intervals on the electromagnet winding current at $\mathrm{C}=5 \mu \mathrm{F} ; \mathrm{u}_{\mathrm{Cav}}=300 \mathrm{~V} ; \Delta \mathrm{u}_{\mathrm{C}}=50 \mathrm{~V} ; \mathrm{R}=20 \mathrm{Ohm}$.

It could be assumed when calculating $t_{\mathrm{ch}}$ and $t_{\text {dis }}$ intervals that current $i_{L}$ changes slowly while its value at the interval $t_{\mathrm{ch}}+t_{\mathrm{dis}}$ is constant being equal to $i_{L \mathrm{Lav}}$ due to the relatively large time constant $T_{L}=L / r$. For the same reason, it could be assumed when calculating current change $i_{L}$ during the process of discharging the energy accumulated in the winding, that current change $i_{L}$ may not depend on voltage ripples at the capacitor and may be determined by average voltage $u_{\text {Cav }}$ which, in our case, remains constant and equal to $u_{\text {Cav }}=\left(U_{\text {th2 }}+U_{\text {th1 } 1}\right) / 2$ during periodic charging-discharging process. Then, current drop $i_{L}$ could be described by the following equation:

$$
i_{L}=\left[i_{L}(0)+\frac{u_{C \mathrm{av}}-E}{r}\right] e^{-t / T_{L}}-\frac{u_{C_{\mathrm{av}}}-E}{r}
$$

where $i_{L}(0)$ is current at the beginning of the drop while $E$ is voltage of the source, switched in series with the electromagnet winding.

We assume that energy transfer to the capacitor during a cyclic process may occur up to some low value of $\alpha i_{L}(0)$, where $\alpha<1$.

Assuming that $t=t_{\text {drop }}$ in Equation (6), we obtain the following:

$$
t_{\mathrm{drop}}=T_{L} \ln \frac{u_{\mathrm{Cav}}-E+r i_{L}(0)}{u_{C \mathrm{av}}-E+\alpha r i_{L}(0)} .
$$


The required value of $u_{C a v}$ could be also obtained by setting the value of $t_{\text {drop. }}$

\section{Recommendations on calculating the power part of the electromagnet current switching unit}

The procedure for calculating the parameters of the power part of the energy discharge circuit at a given maximum switching time of the current tdrop may be as follows:

1) Set the average voltage at the capacitor uCav higher than voltage $\mathrm{E}$ while around $200 \div 300 \mathrm{~V}$ lower than the acceptable voltage value at the main transistor VT1.

2) Select a film capacitor with the capacity at which its dimensions are acceptable in terms of its location on the baseplate.

3) Set the acceptable voltage ripple range $\Delta u \approx 50 \mathrm{~V}$ at the capacitor. Then, the voltage ripple amplitude at the capacitor would amount to $\Delta \mathrm{uC} \approx \Delta \mathrm{u} / 2=25 \mathrm{~V}$. Check the acceptability of such a variable component for the selected capacitor.

4) Determine time tdrop of the electromagnet winding current drop down to zero by formula (7).

\section{Experimental results}

\subsection{Modelling of ECCU}

Circuit simulation has been carried out in MatlabSimulink environment with the following initial data: power supply voltage $E=220 \mathrm{~V} ; U_{\mathrm{th} 1}=250 \mathrm{~V} ; U_{\mathrm{th} 2}=300 \mathrm{~V}$; electromagnet coil inductance $L=1 \mathrm{H}$; active electromagnet coil resistance $r=25 \mathrm{Ohm}$; storage capacitor capacity $C=5 \mu \mathrm{F}$; storage capacitor resistance $R=50 \mathrm{Ohm}$. At the beginning of the modeling process, transistor VT1 is switched on, and the electromagnet winding current increases according to the exponential law up to a steady state value. Transistors VT2 and VT3 are switched off. On switching transistor VT1 off and transistor VT2 on, the electromagnet coil current, shortcircuited through VT2, may start charging capacitor C (Figure 4). Transistor VT3 through which the electromagnetic energy may be discharged from the electromagnet winding to a ballasting resistor is switched on when the capacitor voltage $u_{C}$ comes up to the level of $U_{\mathrm{th} 2}=300 \mathrm{~V}$. When level $U_{\mathrm{th} 1}=250 \mathrm{~V}$ may be is achieved, transistor VT3 may be blocked, and the capacitor recharging process may continue. When the coil current reaches zero, transistor VT2 is switched off while transistor VT3 is switched on to discharge the energy remained in storage capacitor to the ballasting resistor. The selected power circuit parameters along with switching thresholds of the high-power transistor VT3 may allow delaying the process of coil current drop in order to minimize instantaneous power output from the ballasting resistor as well as provide a necessary condition for discharging the capacitor.

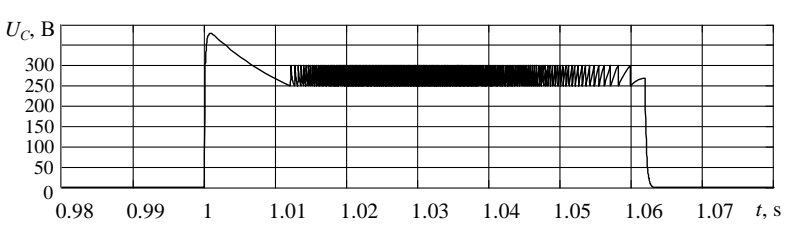

Fig. 4. Voltage at the storage capacitor and instantaneous power output at the resistor at $\mathrm{R}=50 \mathrm{Ohm}, \mathrm{C}=5 \mu \mathrm{F}$.

\subsection{Experimental Verification of ECCU}

Tests have been carried out under normal climate conditions using the installation shown in Figure 5. The installation consists of $3.3 \mathrm{~V}, 5 \mathrm{~V}, 12 \mathrm{~V}$ and 220V DC power supplies, a digital oscilloscope and a magnetic starter of the air switch.

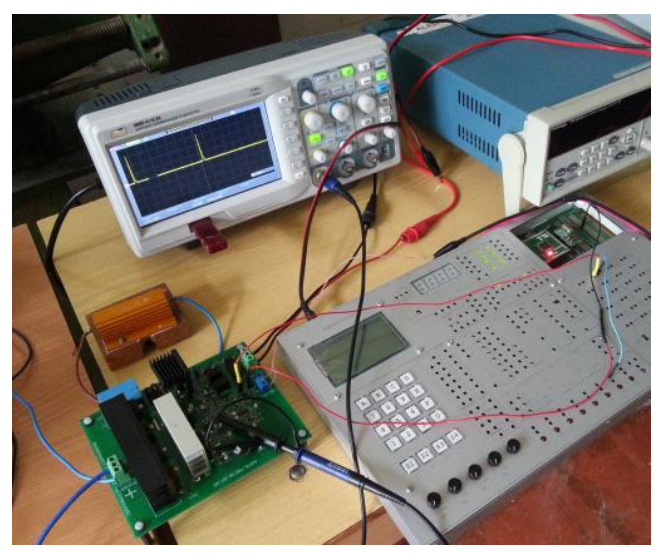

Fig. 5. Experimental installation.

Tests have shown that when the air switch starter of the contactor having inductance of $1 \mathrm{H}$ and current of $6 \mathrm{~A}$ is switched on to provide the electromagnet winding load and current of $6 \mathrm{~A}$, switching-off may take approximately 50ms (Figure 6). Residual voltage at the capacitor may amount to $255 \mathrm{~V}$ with current decrease down to $50 \mathrm{~mA}$ (Figure 7). After switching the control circuit off, the output capacitor is discharged completely in about $500 \mathrm{~ms}$, while it may be discharged down to voltage equal to $10 \%$ of the residual voltage in $350 \mathrm{~ms}$.

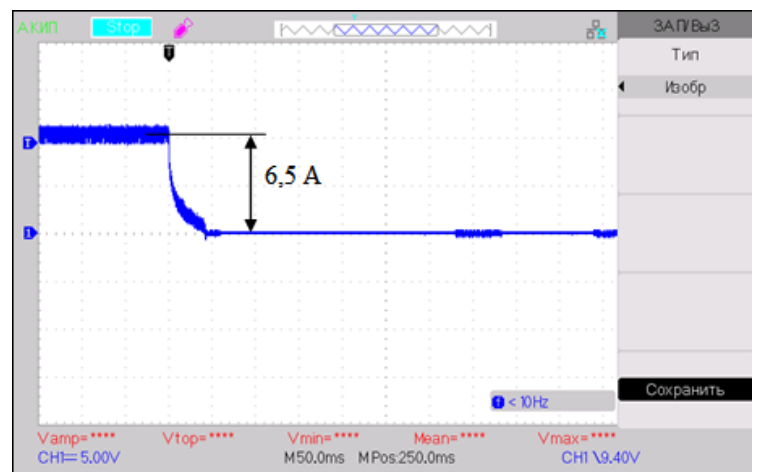

Fig. 6. Oscillograms of current in the starter electromagnet winding. 


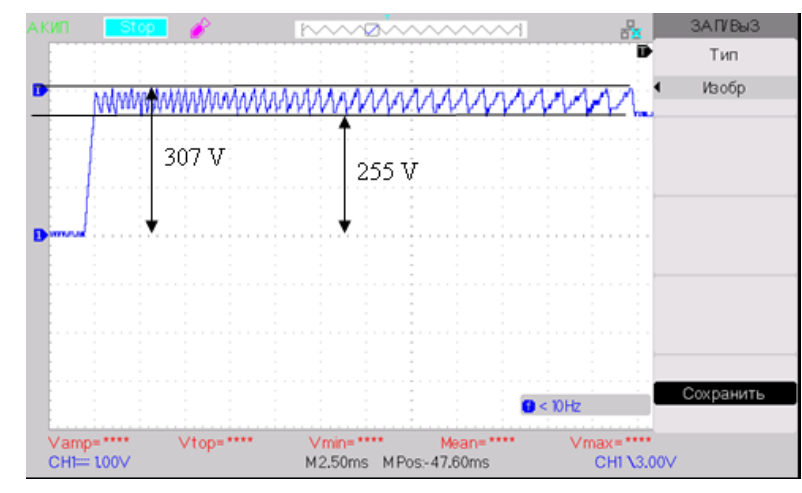

Fig. 7. Oscillograms of voltage at capacitor.

\section{Conclusion}

Thus, fundamental questions concerning the development of the electromagnet current switching unit allowing connecting only two outputs to the circuit consisting of the direct voltage source and the electromagnet winding have been solved in the paper.

\section{References}

[1] A.Yu. Gavrilov, Yu.M. Zaitsev, I.P. Ivanov, V.N. Petrov, G.P. Svintsov, Sposob Upravleniya Elektromagnitnymi Privodami Kontaktorov [The Method of Controlling Electromagnetic Drives of Contactors] Izvestiya vuzov. Elektromekhanika Publ 4(540), 32-37 (2018)

[2] L295 dual switch-mode solenoid driver (SGS-Thomson Microelectronics, http://www.st.com).

[3] ADRV1012K High power solenoid driver (Texas Instruments, https://www.ti.com).

[4] DRV110 120- and $230 \mathrm{VAC}$, 6- to $48 \mathrm{~V} \mathrm{DC}$ current controller for solenoids, relays and valves (Texas Instruments, https://www.ti.com).

[5] VN380 Smart solenoid driver solid state relay (SGS-Thomson Microelectronics, http://www.st.com).

[6] Si7SDOIGD1-120V-20A, Single, 120V 20A Solenoid Driver with Optically Isolated Gate Driver Control Input and with Integrated Finned Heat Sink (Signal Consulting, LLC, http://signalllc.com/Sig_Specs2/Si7FPOexCC1-120V-20A-2TOI1-Spec.pdf).

[7] S. Pithadia Driving solenoid coils efficiently in switchgear applications / Texas Instruments // Analog Application Journal, 4Q, http://www.ti.com. (2013)

[8] Current Controlled Driver for AC Solenoids. TI Designs U584A / Texas Instruments, https://www.ti.com.

[9] J. Van Dam, B. Gysen, M. Dhaens, E. Lomonova, Design, control, and comparison of lowenergy solenoid valve actuators IEEE International Magnetic Conf. 1-2 (2018)

[10] F. He, Y. Zheng, Q. Wang, H. Li, Electromagnetic force control system of solenoidal coil 37th Chinese Control Conf., 8525-8530 (2018)

[11] E. Karaman, A. Mironova, A. Georgiadis, novel actuator approach for workpiece clamping using a pwm driven solenoid IEEE Int. Conf. on Advanced Intelligent Mechatronics 503-508 (2017)

[12] K. Laib, A. Redha Meghnous, M. Tu Pham and X. Lin-Shi, Averaged state mdel and sliding mode for on/off solenoid valve pneumatic actuators American Control Conf. 4569-4574 (2016)

[13] P. Lino, G. Maione, F. Saponaro, J. Deng,K. $\mathrm{Li}$, Identification of solenoid valve dynamics in a variable valve timing system 11th Int. Conf. on Control $1-6(2016)$

[14] F. Meng, G. Tao, P. Luo Pei, Dynamic analysis of proporcional solenoid for automatic transmission application Int. Conf. on Mechatronics and Control 1120-1124 (2014)

[15] C. Sheng-Nian, J. Yu, X. Cheng-Tao, Y. Liang, Q. Ran, Energy-saving driver circuit of highspeed solenoid valve based on soft-switch technology 2nd Int. Conf. on Instrumentation, Measurement, Computer, Communication and Control 598-601 (2012) 